\title{
Intraspecific genotypic richness and relatedness predict the invasibility of microbial communities
}

\author{
Alexandre Jousset ${ }^{1,3}$, Wiebke Schulz ${ }^{1}$, Stefan Scheu ${ }^{1}$ and Nico Eisenhauer ${ }^{1,2,3}$ \\ ${ }^{1}$ Georg-August-University Göttingen, JF Blumenbach Institute of Zoology and Anthropology, Göttingen, \\ Germany and ${ }^{2}$ Department of Forest Resources, University of Minnesota, St. Paul, MN, USA
}

\begin{abstract}
Biological invasions can lead to extinction events in resident communities and compromise ecosystem functioning. We tested the effect of two widespread biodiversity measurements, genotypic richness and genotypic dissimilarity on community invasibility. We manipulated the genetic structure of bacterial communities (Pseudomonas fluorescens) and submitted them to invasion by Serratia liquefaciens. We show that the two diversity measures impact on invasibility via distinct and additive mechanisms. Genotypic dissimilarity of the resident communities linearly increased productivity and in parallel decreased invasion success, indicating that high dissimilarity prevents invasion through niche pre-emption. By contrast, genotypic richness exerted a humpshaped effect on invasion and was linked to the production of toxins antagonistic to the invader. This effect peaked at intermediate richness, suggesting that high richness levels may increase invasibility. Invasibility could be well predicted by the combination of these two mechanisms, documenting that both genotypic richness and dissimilarity need to be considered, if we are to understand the biotic properties determining the susceptibility of ecosystems to biological invasions.
\end{abstract}

The ISME Journal (2011) 5, 1108-1114; doi:10.1038/ismej.2011.9; published online 24 February 2011

Subject Category: microbial population and community ecology

Keywords: Pseudomonas fluorescens; allelopathy; biological invasions; biodiversity-ecosystem functioning relationship; complementarity; niche pre-emption

\section{Introduction}

Human activity has an important role in facilitating biological invasions and is reducing biodiversity worldwide (Vitousek et al., 1996). Invasions of natural communities by foreign species are currently rated as one of the most important global-scale environmental problems (Vitousek et al., 1996; Sala et al., 2000). Biological invasions alter and threaten ecosystem functions by changing the composition of resident communities and the interaction strength between species (Sakai et al., 2001; Sax et al., 2005). Understanding the mechanisms contributing to the resistance of communities against invasions, and driving invader success is essential both for understanding community assembly (Fargione et al., 2003) and for developing conservation strategies.

Biodiversity of the resident community has long been proposed to limit invasibility, but the underlying mechanisms are disputed (Levine and D'Antonio, 1999; Bruno et al., 2003). One commonly accepted mechanism is that diverse communities

Correspondence: A Jousset, Georg-August-University-Göttingen, JF Blumenbach Institute of Zoology and Anthropology, Berliner Street 28, Göttingen 37073, Germany.

E-mail: ajousse@gwdg.de

${ }^{3}$ These two authors contributed equally to the work.

Received 16 September 2010; revised 10 January 2011; accepted 10 January 2011; published online 24 February 2011 are more efficient in resource use, leaving less resources for potential invaders (Hodgson et al., 2002). On the basis of this view stochastic niche theory predicts that low invasibility of diverse communities results from low levels of resources in diverse communities because of stochastic assembly of competitors (Tilman, 2004). Dissimilar communities exploit resources more efficiently and are more productive than simple communities (Heemsbergen et al., 2004; Cadotte et al., 2009). Consequently, habitats colonized by dissimilar communities tend to lack vacant niches (Tilman, 2004) and pose a barrier to invasion (Hodgson et al., 2002). Phylogenetic distance is a good predictor of functional differentiation between organisms (Gardener et al., 2000; Cadotte et al., 2009; Devictor et al., 2010) with intraspecific diversity affecting the functioning of ecosystems to a similar extent or even stronger than species richness (Hughes et al., 2008). Therefore, we hypothesized that intraspecific genotypic dissimilarity of the resident community limits its invasibility.

Rather than on community dissimilarity, other mechanisms are likely to rely more closely on genotypic richness. This diversity index reflects the number of taxa coexisting in a community, and is one of the most prominent diversity measures used in invasion studies (for example, Tilman, 2004; Sax et al., 2005). Richness has an important role in 
the functioning of communities (Bell et al., 2005). Competitive interactions increase with the number of competing taxa (Bell et al., 2009), and they may foster allelopathic interactions, the chemical inhibition of competitors (Dubuis and Haas, 2007, Inglis et al., 2009). Allelopathy is widespread in many systems and has an important role in competitive interactions (Czaran et al., 2002; Validov et al., 2005) and invasion processes (Bais et al., 2003). Hence, we expected that genotypic richness drives the production of toxins inhibiting the invader, thereby reducing invasion success.

To address these issues, we assembled resident model bacterial communities of Pseudomonas fluorescens differing in genotypic dissimilarity and genotypic richness, and subjected them to invasion by Serratia liquefasciens MG1, an unrelated ubiquitous bacterial species with similar niche coverage (Eberl et al., 1996; Schuhegger et al., 2006). Pseudomonas fluorescens is a highly diverse phylogenetic group and a major component of bacterial communities associated with plant roots living on root exudates and producing toxins that inhibit root pathogens (Haas and Keel, 2003), thereby improving plant health. Bacterial communities form an essential component of virtually any ecosystem and drive major ecosystem processes. As bacteria are genetically well characterized, communities assembled from bacterial lineages serve as model systems allowing standardized testing of general ecological hypotheses (Jiang and Morin, 2004; Bell et al., 2005).

We measured the invasibility, productivity and toxicity toward the invader of each community. We tested whether invader success could be predicted by the genotypic dissimilarity and richness of the resident community. Additionally, we tested whether the effects of genotypic dissimilarity and richness could be attributed to changes in the productivity and toxicity of the resident community.

\section{Materials and methods}

The resident bacterial population was built from eight DAPG-producing strains $P$. fluorescens CHA0, PF5, Q2-87, 1M1-96, MVP1-4, F113, Phl1C2 and Q8R1-96, selected in order to cover all major genomic subgroups (de la Fuente et al., 2006). The calculation of the genetic dissimilarity of the populations was carried out in two steps. The genotypic distance between the strains was assessed on the basis of their phlD sequence (see de la Fuente et al. (2006) for details on the phlD sequences of the used strains). This gene is responsible for the synthesis of the polyketide 2, 4-DAPG and is, despite being a functional gene, a robust marker for the phylogenetic affiliation of related strains of DAPG producers (de la Fuente et al., 2006; Moynihan et al., 2009). It is moreover commonly used to describe the genetic structure of environmental communities (Frapolli et al., 2008). The resulting distance matrix was then used to calculate the mean community dissimilarity (Diss) defined as the average distance between the strains (Heemsbergen et al., 2004):

$$
\text { Diss }=\frac{\sum_{i=1}^{n} \sum_{i=j}^{n} G D_{i j}}{\frac{n !}{2 !(n-2) !}}
$$

With $n$ the number of genotypes in the population and GD the genetic distance between pairs of genotypes.

We set up a total of 95 populations covering a richness gradient ranging from one to eight strains (Supplementary Table S1). We used the unrelated bacterium $S$. liquefaciens MG1 as model invader, chromosomally tagged with Green Fluorescent Protein (Eberl et al., 1996).

Bacteria were pre-grown in Luria Bertani broth at $25^{\circ} \mathrm{C}$ for $12 \mathrm{~h}$. Late exponential phase bacteria were pelleted by centrifugation (13k rpm, $1 \mathrm{~min}$ ), washed twice in $1 \% \mathrm{NaCl}$, incubated for $6 \mathrm{~h}$ at room temperature to allow terminating division cycles and adjusted to an $\mathrm{OD}_{600}$ of 1.0. Bacteria were grown in $150 \mu \mathrm{l} \quad 1 / 10 \mathrm{LB}$ medium (Start $\mathrm{OD}_{600}=0.2$ ) supplemented with $S$. liquefaciens $\left(\mathrm{OD}_{600}=0.01\right)$ in 96-wells microtiter plates at $25^{\circ} \mathrm{C}$ with agitation. After $36 \mathrm{~h}$, the density of total bacteria and invaders was quantified with a C6 flow cytometer (Accuri, Ann Harbor, MI, USA). Total bacterial populations were gated on the basis of the forward scatter (FSC) and side scatter (SSC) signals, invaders were gated on the basis of the FL1-A signal (green fluorescence). Invader success was characterized as its relative abundance (percentage of total bacteria) in the population after $36 \mathrm{~h}$, that is, after $\sim 30-40$ generations.

Community productivity was defined as the density of the resident community grown as described above, but without invaders. The production of toxins antagonistic to $S$. liquefaciens by the resident communities was assessed with an agar overlay assay (Parret et al., 2005; Validov et al., 2005). Briefly, a total of 24 communities of P. fluorescens of increasing genotype richness (1, 2, 4 and 8 genotypes, see Supplementary Table S2) were assembled. Drops $(10 \mu \mathrm{l})$ of each community $\left(\mathrm{OD}_{600}=0.1\right)$ were spotted on LB plates and incubated at $25{ }^{\circ} \mathrm{C}$ for $48 \mathrm{~h}$. Bacteria were killed by chloroform fumigation, and the plates covered with an overlay of $S$. liquefaciens $\left(\mathrm{OD}_{600}=0.1\right)$ were embedded in soft agar $(0.5 \%$ Agar in phosphatebuffered saline buffer). Plates were subsequently incubated at $25{ }^{\circ} \mathrm{C}$ for $24 \mathrm{~h}$. Toxin production was assessed as the surface of the inhibition halo, a measure correlated with toxin concentration (Delignette-Muller and Flandrois, 1994). The overlay was applied directly after fumigation to prevent the diffusion of intracellular compounds that may have been released by the fumigation. 
The dependent variables, invader success and community productivity were log-transformed and analyzed with general linear models. We analyzed the effects of genotypic richness, dissimilarity and composition of the resident $P$. fluorescens community on invader success. For analyzing the effect of composition, bacterial communities containing the same species were coded with the same number to avoid pseudo-replication (Supplementary Tables S1 and S2) (Schmid et al., 2002). Significance was assessed with sequential (type I) sums of squares (Schmid et al., 2002). Composition was tested against the residuals, whereas genotypic richness and dissimilarity were tested against composition to account for the fact that (i) each diversity treatment was represented by different species compositions (that is, monocultures to seven-species treatments), and (ii) the eight-species treatment always contained the same species combinations and thus was pseudo-replicated (Huston, 1997; Schmid et al., 2002). Moreover, we investigated whether diversity effects were only due to the presence of single genotypes by fitting each genotype before genotypic diversity measures in separate sequential analyses (Table 1). If fitting single genotypes before genotypic diversity measures removed the effect of the diversity indices, the observed diversity effects may have mainly been due to the inclusion of a dominant genotype in the community (sampling effect; Huston, 1997). Thereby, presence of single strain was tested against composition. We explored the effect of genetic richness and dissimilarity on invader success by fitting parameters with three different models: categorical (ANOVA), linear regression (GLM) and quadratic regression (GLM). The optimal model was selected based on the Akaike Information Criterion (AIC). Before quadratic regression analysis, we carried out a Mitchell-Olds \& Shaw test to ensure that the invasion minimum was within the range of the tested data (Mitchell-Olds and Shaw, 1987).

By sequential parameter fitting we explored two mechanisms, which we hypothesized to be responsible for the relationship between resident community structure and invader success; the increase in resource pre-emption and the increase in antagonistic (allelopathic) interactions with community diversity. According to our hypothesis, if genotypic dissimilarity restricts invader success via resource pre-emption, fitting community productivity before diversity measures should decrease the significance of genotypic dissimilarity.

The effects of the presence of single genotypes, genotypic richness, genotypic dissimilarity and community composition on community productivity and $S$. liquefaciens were tested with sequential GLM as explained above. Additionally, we verified the role of community productivity and toxicity on invasion by fitting the invader success as a function of the community productivity and the predicted toxin production using a GLM (type III SS).

\section{Results}

The two diversity measures, genotypic richness and dissimilarity of the resident $P$. fluorescens community, strongly affected the performance of the invader S. liquefaciens. Genotypic dissimilarity was the major determinant of invader success $\left(\mathrm{F}_{1,67}=53.23, P<0.0001\right)$, and the performance of $S$. liquefaciens decreased linearly with increasing genotypic dissimilarity (Figure 1a). This decrease in invasion success occurred in parallel to an increase in productivity of the resident bacterial community with genotypic dissimilarity $\left(\mathrm{F}_{1,67}=50.04\right.$, $P<0.0001$ and $F_{1,67}=21.28, P<0.0001$ when fitted before and after genotypic richness, respectively; Figure 1b), suggesting that resident communities more efficiently exploiting resources were less vulnerable to invasion.

Genotypic richness also had a strong effect on invader success, and this effect was best described with a quadratic regression $\left(\mathrm{F}_{1,73}=45.51, P<0.0001\right.$ and $F_{1,73}=15.84, P=0.0002$ for the quadratic term

Table 1 Analysis of variance table of F-values on the effects of presence of single genotypes (Genotype; MVP 1-4, Q2-87, CHA0, F113, Phl1C2, PF-5, 1M1-96 and Q8R1-96), GeR and GeD of Pseudomonas fluorescens communities on the success of invasion by Serratia liquefaciens

\begin{tabular}{|c|c|c|c|c|c|c|c|c|c|c|c|c|c|c|c|c|c|}
\hline \multirow{2}{*}{$\begin{array}{l}\text { Factor } \\
\text { Genotype }\end{array}$} & \multirow{2}{*}{$\frac{d f}{1}$} & \multicolumn{2}{|c|}{$M V P 1-4$} & \multicolumn{2}{|c|}{ Q2-87 } & \multicolumn{2}{|c|}{ СHAO } & \multicolumn{2}{|c|}{$F 113$} & \multicolumn{2}{|c|}{ Phl1C2 } & \multicolumn{2}{|c|}{$P F-5$} & \multicolumn{2}{|c|}{$1 M 1-96$} & \multicolumn{2}{|c|}{ Q8R1-96 } \\
\hline & & 10.94 & $* * \downarrow$ & 4.87 & ${ }^{*} \downarrow$ & 14.48 & $* * * \downarrow$ & 6.34 & ${ }^{*} \downarrow$ & 4.33 & ${ }^{*} \downarrow$ & 16.76 & $* * * \downarrow$ & 8.84 & $* * \downarrow$ & 1.71 & Ns \\
\hline & 7 & & & & & & & & & & & & & & & & \\
\hline 1st & & 9.20 & $* * *$ & 10.28 & $* * *$ & 8.80 & $* * *$ & 9.77 & $* * *$ & 10.13 & $* * *$ & 8.71 & $* * *$ & 9.40 & $* * *$ & 11.03 & $* * *$ \\
\hline 2nd & & 4.06 & $* * *$ & 4.72 & $* * *$ & 4.44 & $* * *$ & 3.80 & $* *$ & 4.14 & $* * *$ & 4.34 & $* * *$ & 4.13 & $* * *$ & 4.58 & $* * *$ \\
\hline GeD & 1 & & & & & & & & & & & & & & & & \\
\hline 1st & & 45.03 & $* * *$ & 48.59 & $* * *$ & 38.12 & $* * *$ & 51.76 & $* * *$ & 50.32 & $* * *$ & 37.55 & $* * *$ & 45.92 & $* * *$ & $\mathbf{5 2 . 1 7}$ & $* * *$ \\
\hline 2nd & & 9.05 & $* *$ & 9.70 & $* *$ & 7.63 & $* *$ & 9.93 & $* *$ & 8.42 & $* *$ & 6.92 & * & 9.03 & $* *$ & 6.98 & * \\
\hline Composition & 66 & 0.64 & Ns & 0.63 & Ns & 0.64 & Ns & 0.63 & Ns & 0.64 & Ns & 0.64 & Ns & 0.64 & Ns & 0.63 & Ns \\
\hline Error & 19 & & & & & & & & & & & & & & & & \\
\hline
\end{tabular}

Abbreviations: df, degrees of freedom; GeR, genotypic richness; GeD, genotypic dissimilarity; Ns, not significant.

In separate sequential analyses each of the genotypes was fitted before fitting genotypic richness and genotypic dissimilarity, with the latter two fitted before (1st) and after (2nd) the respective other.

${ }^{* *} P<0.001,{ }^{*} P<0.01,{ }^{*} P<0.05$, Ns $(P>0.05), \downarrow=$ decrease in invasion success of Serratia liquefaciens. Significant effects are given in bold. 

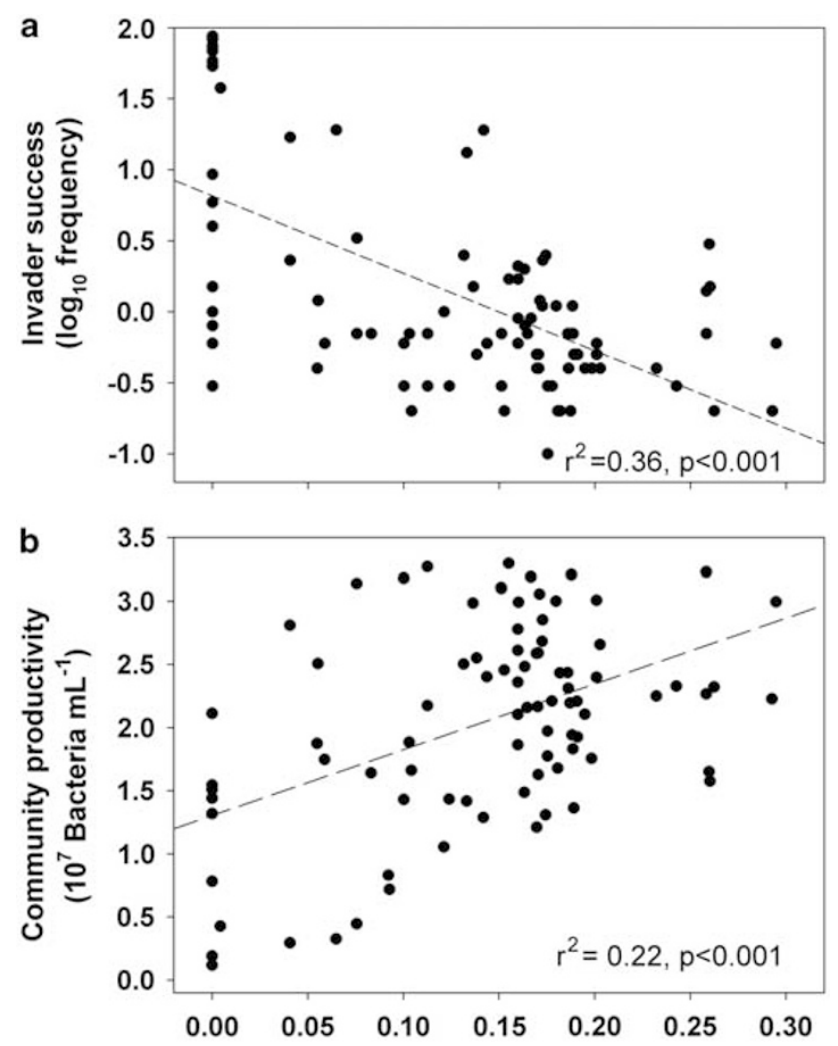

Genotypic dissimilarity resident community

Figure 1 Effect of intraspecific genotypic dissimilarity on the invasion success of Serratia liquefaciens (a) and on the performance of Pseudomonas fluorescens communities assembled from one to eight different strains without the invader (b). Productivity was defined as the bacterial concentration, invader success as its relative abundance (log transformed) in the population at the end of the experiment (i.e., after 30-40 generations).

of genotypic richness when fitted before and after genotypic dissimilarity, respectively). Invader success was lowest at a genotypic richness of $5.03 \pm 0.22$ (Mitchell-Olds and Shaw test, Figure 2a). This pattern mirrored the production of toxins by the resident community; the inhibition of $S$. liquefaciens in the agar overlay assay depended on genotypic richness and peaked at intermediate richness (Figure 2b). Genotypic richness exerted a hump-shaped effect on the size of the inhibition halo (Figure 2b; $\mathrm{F}_{1,19}=14.4, \quad P=0.0012$ and $\mathrm{F}_{1,19}=16.93, P=0.0006$ for the linear and quadratic term of richness, fitted after PF-5). By contrast, genotypic dissimilarity had no significant impact on toxin production $\left(\mathrm{F}_{1,19}=3.21, P=0.09\right)$. Moreover, communities containing the strain PF5 increased the inhibition of the invader $\left(\mathrm{F}_{1,19}=28.11\right.$, $P<0.0001$ ).

As given above, fitting genotypic richness first did not eliminate the significant effect of dissimilarity on invasion success and vice versa, indicating that the two diversity measures explained different components of the variance (Schmid et al., 2002).
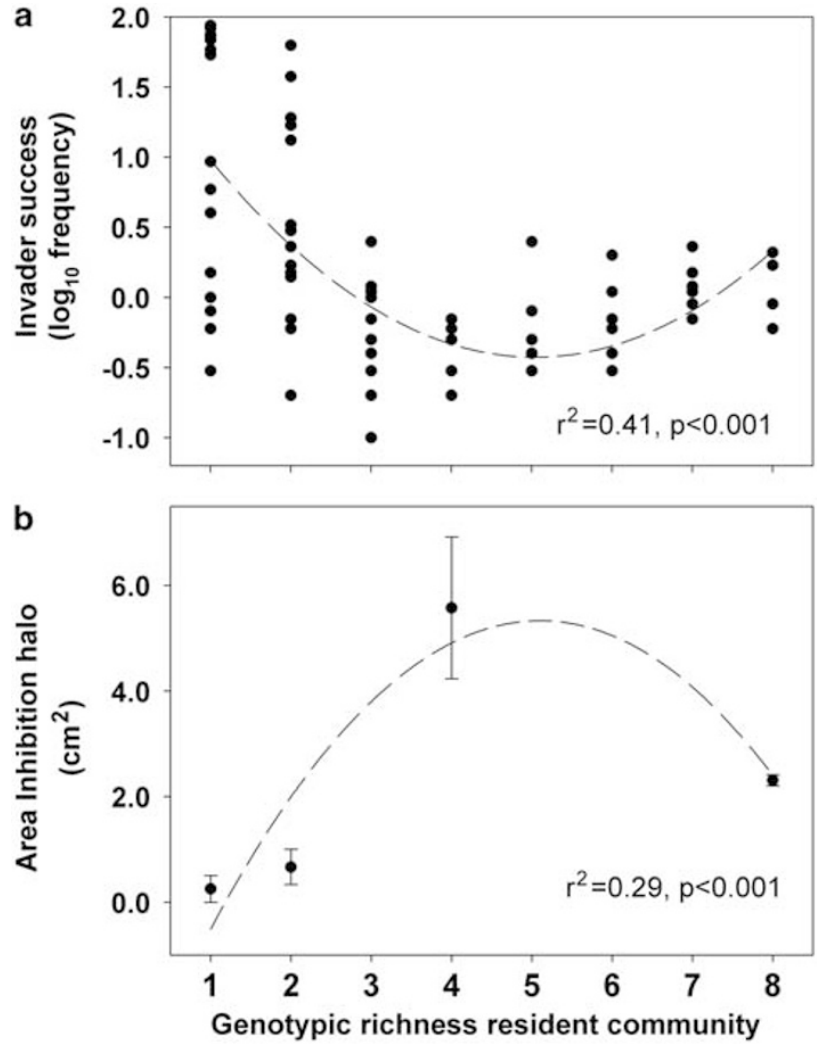

Figure 2 Effect of intraspecific genotypic richness of communities of Pseudomonas fluorescens on the invasion success of Serratia liquefaciens (a) and on the production of toxins inhibiting the invader strain $S$. liquefaciens (b). Toxicity is expressed as area of the inhibition halo around chloroform-killed colonies of $P$. fluorescens overlaid with $S$. liquefaciens. The dashed line shows a simple quadratic fit, see Results for complete model and significance values. Invader success is expressed as its relative abundance (log transformed) in the population at the end of the experiment (i.e., after 30-40 generations).

Moreover, the effects of genotypic richness and dissimilarity remained highly significant even when fitted after single bacterial genotypes (Table 1), indicating that both diversity effects did not only rely on the presence of certain dominant genotypes, although all genotypes with the exception of Q8R1-96 in the resident bacterial community decreased invasion success of $S$. liquefaciens. Additionally, fitting community productivity as covariate in separate sequential ANCOVAs revealed a strong negative correlation between invasion success and productivity of the resident community $\left(\mathrm{F}_{1,73}=228.55, \quad P<0.0001\right)$. Fitting community productivity before genotypic diversity measures decreased particularly the significance of genotypic dissimilarity $\left(\mathrm{F}_{1,72}=7.21, \quad P=0.0089\right.$ and $\mathrm{F}_{1,72}=1.98, P=0.1640$ when fitted before and after genotypic richness, respectively) and much less that of genotypic richness $\left(\mathrm{F}_{1,72}=16.12, P=0.0001\right.$ and $\mathrm{F}_{1,72}=10.88, P=0.0015$ when fitted before and after genotypic dissimilarity, respectively), suggesting 
that effects of genotypic dissimilarity can be attributed primarily to community productivity.

The invasibility could be well predicted $\left(R^{2}=0.57\right)$ as a function of the productivity $\left(\mathrm{F}_{1,83}=46.77, P<0.0001\right)$ and toxicity $\left(\mathrm{F}_{1,83}=20.02\right.$, $P<0.0001)$ of the resident community, confirming that these two mechanisms function as major determinants of community invasibility.

\section{Discussion}

Invasion poses a major threat to the diversity and functioning of ecosystems, and resistance of communities has been suggested to rely on a complex interplay between abiotic and biotic factors (Hooper et al., 2005). This study dissected the effects of different diversity measures on community invasibility and revealed two distinct mechanisms linking biodiversity and invasibility of microbial communities. Genetically dissimilar communities performed better and were less susceptible to invasion, suggesting that they used resources more efficiently resulting in niche pre-emption. By contrast, genotypic richness determined the production of toxins inhibiting the invader. These two effects were largely independent from each other and likely affected invasion success in an additive way.

Genotypic dissimilarity of the resident community was the most important feature driving invasion success in this study. The success of the invader decreased linearly with increasing dissimilarity of the resident community and this effect was linked to the higher productivity of dissimilar communities. This indicates that the performance of the resident community is a major factor affecting its invasibility. Phylogenetic distance between $P$. fluorescens lineages correlates well with their metabolic specialization and resource use (Gardener et al., 2000). Dissimilar communities likely cover more niches leaving less resources for the invader (Tilman, 2004). This adds to the increasing evidence that functional dissimilarity of species drives essential ecosystem functions (Heemsbergen et al., 2004; Cadotte et al., 2009; Hillebrand and Matthiessen, 2009) and highlights that this effect not only occurs at the species level, but also at the level of genotypes within species. The rapid genetic diversification in bacterial populations (Boles et al., 2004) may thus contribute to reduce invasibility, and reported negative relationships between community productivity and invasibility (Hodgson et al., 2002) are probably mainly based on niche pre-emption of dissimilar communities (Tilman, 2004; Fargione and Tilman, 2005). Our findings highlight the role of genotypic dissimilarity in invasibility and contrast the results by Hodgson et al. (2002) suggesting that invasibility of bacterial communities is mainly governed by the presence of dominant genotypes.

Genotypic richness of the resident community was the second major determinant of invader success and functioned by driving the production of antagonistic toxins. Most tested genotypes inhibited the invader, and one genotype, PF-5, was particularly aggressive. Toxin production presented a hump-shaped pattern and peaked at intermediate richness, thereby contributing to prevent invader establishment. Bacteria can sense competitors and react by overproducing toxins (Dubuis and Haas, 2007). Most lineages of $P$. fluorescens produce allelopathic toxins such as strain-specific bacteriocins (Validov et al., 2005) and broad-spectrum antibiotics like 2,4-DAPG or hydrogen cyanide (Haas and Keel, 2003). Strong competition, as occurs within diverse communities (Bell et al., 2009), favors allelopathic strains (Inglis et al., 2009). Increased antagonistic interactions within the resident communities therefore likely contributed to the elevated community resistance against the invading S. liquefaciens. The decline in invader resistance at higher genotypic richness may have been due to the decline in the strength of pairwise interactions relaxing allelopathy, or to an excessive selfpoisoning of the resident community. Allelopathic interactions, where organisms poison competitors, are common in terrestrial and marine ecosystems (Jackson and Buss, 1975; Wardle et al., 1996). Our results indicate that allelopathic interactions should be considered as potential mechanism determining community invasibility.

In conclusion, we showed that the genetic structure of a community affects invader success by determining available resources and allelopathic interactions. As these two factors form major regulatory forces in various ecosystems, both genotypic dissimilarity and richness need to be considered, if we are to understand the mechanisms responsible for the susceptibility of ecological systems against major global threats such as biological invasions. Considering both effects in a single model may allow explaining diverging observations on the effect of biodiversity on invasibility and improve predictive models on biological invasions.

\section{Acknowledgements}

We are grateful to Linda Thomashow (USDA-ARS) for providing the $P$. fluorescens strains Q2-87, Phl1C2, 1M196, Q8R1-96 and MVP1-4, Rafael Rivilla (Universidad autónoma de Madrid) for providing P. fluorescens F113 and Anton Hartmann (Helmholtz Zentrum München) for providing Serratia liquefaciens MG1. Thanks to Jan Schrader for his assistance with flow cytometry and to Bernhard Schmid (University of Zurich) for valuable comments on the statistical analyses.

\section{References}

Bais HP, Vepachedu R, Gilroy S, Callaway RM, Vivanco JM. (2003). Allelopathy and exotic plant invasion: from molecules and genes to species interactions. Science 301: 1377-1380. 
Bell T, Lilley AK, Hector A, Schmid B, King L, Newman JA. (2009). A linear model method for biodiversityecosystem functioning experiments. Am Nat 174: 836-849.

Bell T, Newman JA, Silverman BW, Turner SL, Lilley AK. (2005). The contribution of species richness and composition to bacterial services. Nature 436: 1157-1160.

Boles BR, Thoendel M, Singh PK. (2004). Self-generated diversity produces 'insurance effects' in biofilm communities. Proc Natl Acad Sci USA 101: 16630-16635.

Bruno JF, Stachowicz JJ, Bertness MD. (2003). Inclusion of facilitation into ecological theory. Trends Ecol Evol 18: $119-125$.

Cadotte MW, Cavender-Bares J, Tilman D, Oakley TH. (2009). Using phylogenetic, functional and trait diversity to understand patterns of plant community productivity. PLoS ONE 4: e5695.

Czaran TL, Hoekstra RF, Pagie L. (2002). Chemical warfare between microbes promotes biodiversity. Proc Natl Acad Sci USA 99: 786-790.

de la Fuente L, Mavrodi DV, Landa BB, Thomashow LS, Weller DM. (2006). phlD-based genetic diversity and detection of genotypes of 2,4-diacetylphloroglucinolproducing Pseudomonas fluorescens. FEMS Microbiol Ecol 56: 64-78.

Delignette-Muller ML, Flandrois JP. (1994). An accurate diffusion method for determining bacterial sensitivity to antibiotics. J Antimicrob Chemother 34: 73-81.

Devictor V, Mouillot D, Meynard C, Jiguet F, Thuiller W, Mouquet N. (2010). Spatial mismatch and congruence between taxonomic, phylogenetic and functional diversity: the need for integrative conservation strategies in a changing world. Ecol Lett 13: 1030-1040.

Dubuis C, Haas D. (2007). Cross-species GacA-controlled induction of antibiosis in pseudomonads. Appl Environ Microbiol 73: 650-654.

Eberl L, Winson MK, Sternberg C, Stewart GSAB, Christiansen G, Chhabra SR et al. (1996). Involvement of N-acyl-L-homoserine lactone autoinducers in controlling the multicellular behaviour of Serratia liquefaciens. Mol Microbiol 20: 127-136.

Fargione J, Brown CS, Tilman D. (2003). Community assembly and invasion: An experimental test of neutral versus niche processes. Proc Natl Acad Sci USA 100: 8916-8920.

Fargione JE, Tilman D. (2005). Diversity decreases invasion via both sampling and complementarity effects. Ecol Lett 8: 604-611.

Frapolli M, Moënne-Loccoz Y, Meyer J, Défago G. (2008). A new DGGE protocol targeting 2,4-diacetylphloroglucinol biosynthetic gene phlD from phylogenetically contrasted biocontrol pseudomonads for assessment of disease-suppressive soils. FEMS Microbiol Ecol 64: 468-481.

Gardener BBM, Schroeder KL, Kalloger SE, Raaijmakers JM, Thomashow LS, Weller DM. (2000). Genotypic and phenotypic diversity of phlD-containing Pseudomonas strains isolated from the rhizosphere of wheat. Appl Environ Microbiol 66: 1939-1946.

Haas D, Keel C. (2003). Regulation of antibiotic production in root-colonizing Pseudomonas spp.and relevance for biological control of plant disease. Annu Rev Phytopathol 41: 117-153.

Heemsbergen DA, Berg MP, Loreau M, van Haj JR, Faber JH, Verhoef HA. (2004). Biodiversity effects on soil processes explained by interspecific functional dissimilarity. Science 306: 1019-1020.

Hillebrand H, Matthiessen B. (2009). Biodiversity in a complex world: consolidation and progress in functional biodiversity research. Ecol Lett 12: 1405-1419.

Hodgson DJ, Rainey PB, Buckling A. (2002). Mechanisms linking diversity, productivity and invasibility in experimental bacterial communities. Proc $R$ Soc B-Biol Sci 269: 2277-2283.

Hooper DU, Chapin FS, Ewel JJ, Hector A, Inchausti P, Lavorel S et al. (2005). Effects of biodiversity on ecosystem functioning: A consensus of current knowledge. Ecol Monogr 75: 3-35.

Hughes AR, Inouye BD, Johnson MTJ, Underwood N, Vellend M. (2008). Ecological consequences of genetic diversity. Ecol Lett 11: 609-623.

Huston MA. (1997). Hidden treatments in ecological experiments: Re-evaluating the ecosystem function of biodiversity. Oecologia 110: 449-460.

Inglis RF, Gardner A, Cornelis P, Buckling A. (2009). Spite and virulence in the bacterium Pseudomonas aeruginosa. Proc Natl Acad Sci USA 106: 5703-5707.

Jackson JBC, Buss L. (1975). Allelopathy and spatial competition among coral-reef invertebrates. Proc Natl Acad Sci USA 72: 5160-5163.

Jiang L, Morin PJ. (2004). Productivity gradients cause positive diversity-invasibility relationships in microbial communities. Ecol Lett 7: 1047-1057.

Levine JM, D'Antonio CM. (1999). Elton revisited: a review of evidence linking diversity and invasibility. Oikos 87: 15-26.

Mitchell-Olds T, Shaw RG. (1987). Regression analysis of natural selection - statistical inference and biological interpretation. Evolution 41: 1149-1161.

Moynihan JA, Morrissey JP, Coppoolse ER, Stiekema WJ, O'Gara F, Boyd EF. (2009). Evolutionary history of the phl gene cluster in the plant-associated bacterium Pseudomonas fluorescens. Appl Environ Microbiol 75: 2122-2131.

Parret AHA, Temmerman K, De Mot R. (2005). Novel lectin-like bacteriocins of biocontrol strain Pseudomonas fluorescens Pf-5. Appl Environ Microbiol 71: 5197-5207.

Sakai AK, Allendorf FW, Holt JS, Lodge DM, Molofsky J, With KA et al. (2001). The population biology of invasive species. Annu Rev Ecol Syst 32: 305-332.

Sala OE, Chapin FS, Armesto JJ, Berlow E, Bloomfield J, Dirzo R et al. (2000). Biodiversity-Global biodiversity scenarios for the year 2100. Science 287: 1770-1774.

Sax DF, Kinlan BP, Smith KF. (2005). A conceptual framework for comparing species assemblages in native and exotic habitats. Oikos 108: 457-464.

Schmid B, Hector A, Huston MA, Inchausti P, Nijs I, Leadley PW et al. (2002). The design and analysis of biodiversity experiments. In: Loreau $\mathrm{M}$, Naeem S and Inchausti P (eds). Biodiversity and Ecosystem Functioning: Synthesis and Perspectives. Oxford University Press: New York.

Schuhegger R, Ihring A, Gantner S, Bahnweg G, Knappe C, Vogg $G$ et al. (2006). Induction of systemic resistance in tomato by N-acyl-L-homoserine lactone-producing rhizosphere bacteria. Plant Cell Environ 29: 909-918. 
Tilman D. (2004). Niche tradeoffs, neutrality, and community structure: a stochastic theory of resource competition, invasion, and community assembly. Proc Natl Acad Sci USA 101: 10854-10861.

Validov S, Mavrodi O, De La Fuente L, Boronin A, Weller D, Thomashow L et al. (2005). Antagonistic activity among 2,4-diacetylphloroglucinol-producing fluorescent Pseudomonas spp. FEMS Microbiol Letters 242: 249-256.
Vitousek PM, DAntonio CM, Loope LL, Westbrooks R. (1996). Biological invasions as global environmental change. American Scientist 84: 468-478.

Wardle DA, Nicholson KS, Rahman A. (1996). Use of a comparative approach to identify allelopathic potential and relationship between allelopathy bioassays and "competition" experiments for ten grassland and plant species. J Chem Ecol 22: 933-948.

Supplementary Information accompanies the paper on The ISME Journal website (http://www.nature.com/ismej) 